\title{
TWO DEFINITIONS OF CONNECTIONS ON MANIFOLDS AND OPERATIONS ON THE DIFFERENTIAL FORMS IN LOCAL COORDINATES
}

\author{
MIROSLAV KUREŠ \\ Institute of Mathematics, Brno University of Technology, Brno, Czech Republic \\ Email Address: kures@fme.vutbr.cz
}

Received March 19, 2019

\begin{abstract}
Aвstract. Some important concepts of differential geometry are calculated in local coordinates. In particular, two definition of a connection are compared. Furthermore, the paper also deals with coordinate expression of differential forms.
\end{abstract}

2010 Mathematics Subject Classification. 53C05, 58A05, 58A10, 58A20.

Key words and phrases. connections on manifolds; local coordinates; Hodge star operator.

\section{INTRODUCTION}

The objective of this paper is twofold. First, give a clear coordinate representation of connections called the Ehresmann connection or the nonlinear connection; to clarify two dual definitions of such connections, especially the jet definition, with respect to various applications, e.g. in relativistic electromagnetism, see [1]. Second, the paper should help to illustrate the concepts of differential geometry in examples. It should also be an inspiration for other examples. Differential geometry is demanding for abstraction, and in many common textbooks there is "no place" to demonstrate calculations in local coordinates. In the paper, we are trying to replace this gap and be a tool for both teachers and students.

\section{An example of a manifold Without additional StRUCtURes but With a CONNECTION}

1.1. A manifold and charts. Let $M$ be an infinite (circular) cylinder and let the cylinder has a tangent sphere in every its point. We consider the set of all these spheres as the total manifold $Y$ fibered over $M$ with every such a sphere as a fiber $F$. We write $\pi: Y \rightarrow M$ ( $\pi$ standing for the projection).

The base manifold $M$ is two-dimensional, but it cannot be covered by a single chart. The case of the cylinder is the same as the case of the circle. Two charts are sufficient, but four charts are more comfortable. We denote the local coordinates by $(x, y)$.

DOI: $10.28924 /$ APJM/6-11 


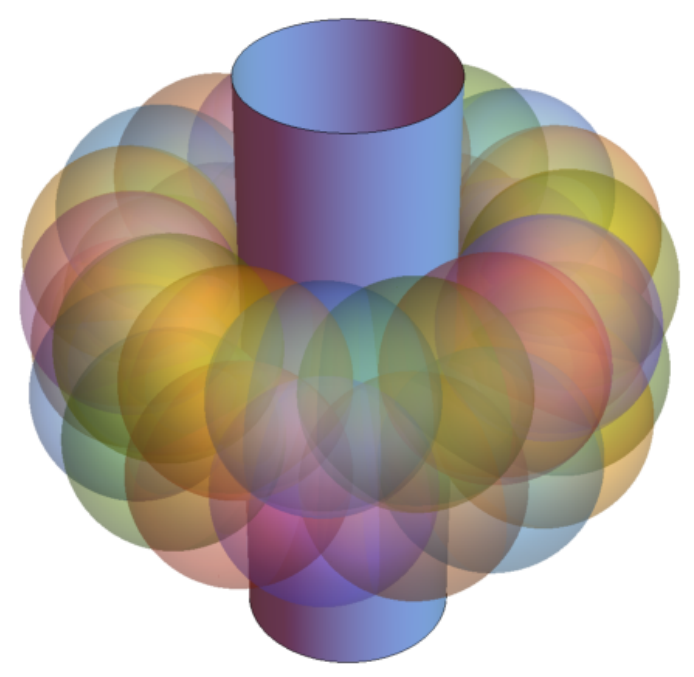

FIGURE 1. Spheres tangent to the cylinder.

1.2. An action on fibers. Similarly, the sphere cannot be covered case by a single chart, too. As for the circle, one may define one chart that covers the whole sphere excluding one point. Thus two charts (forming socalled stereographic atlas) are sufficient. However, the sphere can be covered by an atlas of six charts by more comfortable way: by the projection pointing up, down, left, right, forward and backward. We denote the local coordinates by $(u, v)$. So, the local coordinates of $Y$ are $(x, y, u, v)$. In fact, we are considering the disjoint union of all spheres. Of course, the four-dimensional manifold $Y$ cannot be perfectly represented in the three-dimensional space; so we hope that our Figure 1 is not very confusing.

For an action on two-dimensional sphere, we consider continuous subgroups of the special orthogonal group $\mathrm{SO}(3)$ for a classification of all subgroups of $\mathrm{SO}(3)$ and their symmetry classes see e.g. [3].

We will consider the circle group $U(1)=\{\zeta \in \mathbb{C} ;|\zeta|=1\}$ (where the group operation is the addition of angle $\theta \in[0,2 \pi]$ in $\zeta=\mathrm{e}^{\mathrm{i} \theta}=\cos \theta+\mathrm{i} \sin \theta$ ) which acts on $\mathbb{R}^{3}$ (and hence on $S^{2}$ ) by

$$
\begin{aligned}
& \left(\text { action }[0,2 \pi) \times \mathbb{R}^{3} \rightarrow \mathbb{R}^{3}\right) \\
& \qquad(x, y, z) e^{\mathrm{i} \theta}=(x \cos \theta+y \sin \theta,-x \sin \theta+y \cos \theta, z) .
\end{aligned}
$$

We call the point in which a sphere touches the cylinder the North Pole and its opposite point the South Pole. So the action of the group $U(1)$ means a rotation (which is clockwise with increasing $\theta$ ) under which the North Pole and the South Pole remain fixed.

Using a suitable chart, we have this expression of the action

$$
\begin{aligned}
& \left(\text { action }[0,2 \pi) \times \mathbb{R}^{2} \rightarrow \mathbb{R}^{2}\right) \\
& \qquad(x, z) e^{\mathrm{i} \theta}=\left(x \cos \theta+\sqrt{1-x^{2}-z^{2}} \sin \theta, z\right) .
\end{aligned}
$$

Of course, when using local coordinates, one also need to specify which chart has in mind. 


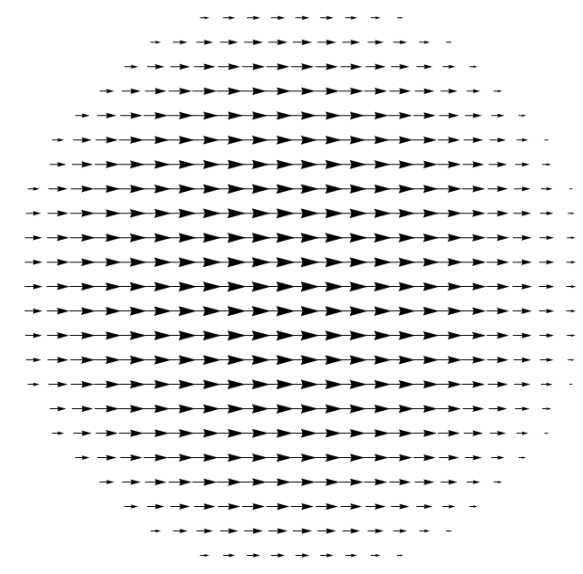

FIGURE 2. Tangent field to the action, $\theta=\frac{\pi}{24}$.

1.3. Tangent bundles of base and fibered manifolds. Tangent bundle $T M$ has local coordinates ( $x, y, X, Y)$; we obtain new coordinates $X, Y$ by the following way. For a point $\left(x_{0}, y_{0}\right)$ we consider a curve $c: \mathbb{R} \rightarrow M$. i.e. $t \mapsto(x(t), y(t))$ such that $\left(x\left(t_{0}\right), y\left(t_{0}\right)\right)=\left(x_{0}, y_{0}\right)$. Then $\left(\frac{\mathrm{d} x(t)}{\mathrm{d} t}\left(t_{0}\right), \frac{\mathrm{d} y(t)}{\mathrm{d} t}\left(t_{0}\right)\right)$ is denoted by $(X, Y)$. Analogously, $T Y$ has local coordinates $(x, y, u, v, X, Y, U, V)$.

The induced projection $T \pi: T Y \rightarrow T M$ works by

$$
T \pi((x, y, u, v, X, Y, U, V))=(x, y, X, Y) .
$$

The vertical bundle $V Y$ has local coordinates $(x, y, u, v, U, V)$ (shortly instead $(x, y, u, v, 0,0, U, V))$.

1.4. Vector and tensor fields. Vector fields on $M$ forming $\mathfrak{X}(M)$ are smooth sections of a form

$$
\begin{aligned}
& X=\xi(x, y) \\
& Y=\psi(x, y)
\end{aligned}
$$

and vector fields on $Y$ forming $\mathfrak{X}(Y)$ are smooth sections of a form

$$
\begin{aligned}
X & =\bar{\xi}(x, y, u, v) \\
Y & =\bar{\psi}(x, y, u, v) \\
U & =\bar{v}(x, y, u, v) \\
V & =\bar{\omega}(x, y, u, v)
\end{aligned}
$$

and for vertical vector fields on $Y$

$$
\bar{\xi}(x, y, u, v)=\bar{\psi}(x, y, u, v)=0
$$

is satisfied.

1.5. The connection - the first definition. A connection on the fiber bundle $p: Y \rightarrow M$ is a $(1,1)$-tensor field on $Y$ (which can be represented as a linear map $\kappa: T Y \rightarrow T Y$ ) such that $\kappa \circ \kappa=\kappa$ and im $\kappa=V Y$. We start with the 
linear map from $T Y$ to $V Y$

$$
\begin{aligned}
& X=0 \\
& Y=0 \\
& U=A(x, y, u, v) X+B(x, y, u, v) Y+E(x, y, u, v) U+F(x, y, u, v) V \\
& V=C(x, y, u, v) X+D(x, y, u, v) Y+G(x, y, u, v) U+H(x, y, u, v) V
\end{aligned}
$$

and observe that requirements of $\kappa \circ \kappa=\kappa$ and im $\kappa=V Y$ enforce

$$
E(x, y, u, v)=H(x, y, u, v)=1 \text { and } F(x, y, u, v)=G(x, y, u, v)=0 .
$$

Hence $\kappa$ is of a form

$$
\begin{aligned}
U & =U+A(x, y, u, v) X+B(x, y, u, v) Y \\
V & =V+C(x, y, u, v) X+D(x, y, u, v) Y .
\end{aligned}
$$

Vectors lying in ker $\kappa$ are called horizontal vectors. Horizontal vectors form horizontal bundle $H Y$. So we have $\left.\kappa\right|_{V Y}=\operatorname{id}_{V Y}$ and $\left.\kappa\right|_{H Y}=0$ and $T_{p} Y=V_{p} Y \oplus H_{p} Y$ for every point $p=(x, y, u, v)$. Disjoint unions of $V_{p} Y$ and $H_{p} Y$ over all points $p \in Y$ are called vertical and horizontal distributions, respectively. The map $\chi: T Y \rightarrow T Y$ defined by $\chi=\operatorname{id}_{T Y}-\kappa$ is called the horizontal projection and its coordinate expression is

$$
\begin{aligned}
& X=X \\
& Y=Y \\
& U=-A(x, y, u, v) X-B(x, y, u, v) Y \\
& V=-C(x, y, u, v) X-D(x, y, u, v) Y
\end{aligned}
$$

is often used to specify a connection.

1.6. The connection - the second (jet) definition. We present also an alternative approach. The first jet prolongation of $p: Y \rightarrow M$ is given by 1-jets of smooth sections of $Y$. Such sections look like

$$
\begin{aligned}
& u=R(x, y) \\
& v=S(x, y)
\end{aligned}
$$

and 1-jets have a form

$$
\begin{aligned}
U & =\frac{\partial R}{\partial x}(x, y) X+\frac{\partial R}{\partial y}(x, y) Y \\
V & =\frac{\partial S}{\partial x}(x, y) X+\frac{\partial S}{\partial y}(x, y) Y .
\end{aligned}
$$

We denote $R_{1}=\frac{\partial R}{\partial x}(x, y), R_{2}=\frac{\partial R}{\partial y}(x, y), S_{1}=\frac{\partial S}{\partial x}(x, y), S_{2}=\frac{\partial S}{\partial y}(x, y)$, which are coordinates of $J^{1} Y:$ in total, $J^{1} Y$ is fibered over $Y$ and has local coordinates $\left(x, y, u, v, R_{1}, R_{2}, S_{1}, S_{2}\right)$. Sections $Y \rightarrow J^{1} Y$ are of a form 


$$
\begin{aligned}
& R_{1}=\bar{A}(x, y, u, v) \\
& R_{2}=\bar{B}(x, y, u, v) \\
& S_{1}=\bar{C}(x, y, u, v) \\
& S_{2}=\bar{D}(x, y, u, v) .
\end{aligned}
$$

We identify $A=\bar{A}, B=\bar{B}, C=\bar{C}, D=\bar{D}$ (eventually $-A=\bar{A},-B=\bar{B},-C=\bar{C},-D=\bar{D}$ ).

Thus, we see that the connection is given by a determining of four functions (in four variables). If we take all these function zero, we obtain vertical vectors $(0,0, U, V)$ and horizontal vectors $(X, Y, 0,0)$. Nevertheless, it is a local property depending on a chosen chart.

The horizontal lift (with respect to $\kappa$ ) of a vector $w \in T_{p} M$ is the vector $\tilde{w} \in T_{P} Y$ (where $\pi(P)=p$ ) such that $\tilde{w}$ is horizontal (with respect to $\kappa$ ) and $T \pi(\tilde{w})=w$. This can be extended to vector fields.

1.7. The principal connection. Let us consider the principal right action (see [2], 10.2, 11.2) $r^{\theta}: Y \rightarrow Y$ determined by the action on fibers described above. Consider

$$
\operatorname{Tr}^{\theta} \circ \kappa=\kappa \circ \operatorname{Tr}^{\theta}
$$

and compute

$$
\begin{gathered}
r^{\theta}: \quad(x, y, u, v) \mapsto\left(x, y, u \cos \theta+\sqrt{1-u^{2}-v^{2}} \sin \theta, v\right) \\
\operatorname{Tr}^{\theta}: \quad(x, y, u, v, X, Y, U, V) \mapsto \\
\left(x, y, u \cos \theta+\sqrt{1-u^{2}-v^{2}} \sin \theta, v, X, Y,\left(\cos \theta-\frac{u}{\sqrt{1-u^{2}-v^{2}}}\right) U-\frac{v}{\sqrt{1-u^{2}-v^{2}}} V, V\right),
\end{gathered}
$$

in fiber coordinates i.e.

$$
\begin{aligned}
& X=X \\
& Y=Y \\
& U=\left(\cos \theta-\frac{u}{\sqrt{1-u^{2}-v^{2}}}\right) U-\frac{v}{\sqrt{1-u^{2}-v^{2}}} V \\
& V=V
\end{aligned}
$$

and then $\operatorname{Tr}^{\theta} \circ \kappa$ has a form

$$
\begin{aligned}
& X=0 \\
& Y=0 \\
& U=\left(\cos \theta-\frac{u}{\sqrt{1-u^{2}-v^{2}}}\right) U-\frac{v}{\sqrt{1-u^{2}-v^{2}}} V \\
& V=V
\end{aligned}
$$

and $\kappa \circ \operatorname{Tr}^{\theta}$ is of a form 


$$
\begin{aligned}
X & =0 \\
Y & =0 \\
U & =\left(\cos \theta-\frac{u}{\sqrt{1-u^{2}-v^{2}}}\right) U-\frac{v}{\sqrt{1-u^{2}-v^{2}}} V+A(x, y, u, v) X+B(x, y, u, v) Y \\
V & =V+C(x, y, u, v) X+D(x, y, u, v) Y
\end{aligned}
$$

and it follows $A(x, y, u, v)=B(x, y, u, v)=C(x, y, u, v)=D(x, y, u, v)=0$ and that is why the flat connection is the principal connection in our example.

\section{Differential FORMS}

2.1. $k$-forms. In the text, we consider only real vector spaces and real smooth manifolds. The Einstein summation convention is used.

Linear, bilinear, ..., forms are called 1-forms, 2-forms, in general $k$-forms. They are nothing but linear maps (homomorphisms) from the $k$-th Cartesian power of a real vector space $\mathcal{V}$ to $\mathbb{R}$, let us stress that they are linear in all components. We fix the dimension of $\mathcal{V}$ as $n$.

First, let us take a look at 2-forms, for an illustration. Let $\alpha: \mathcal{V} \times \mathcal{V} \rightarrow \mathbb{R}$. We express $\alpha\left(\mathbf{v}_{1}, \mathbf{v}_{2}\right)$ in coordinates by $\alpha_{i j} v_{1}^{i} v_{2}^{j}$. If we take 1 -forms $\mathrm{d} x^{i}$ assigning to a vector its $i$-th components, we can write $\alpha_{i j} \mathrm{~d} x^{i} \mathrm{~d} x^{j}$.

The requirement $\alpha\left(\mathbf{v}_{1}, \mathbf{v}_{2}\right)=\alpha\left(\mathbf{v}_{2}, \mathbf{v}_{1}\right)$ (for all $\mathbf{v}_{1}, \mathbf{v}_{2} \in \mathcal{V}$ ) means $\alpha_{i j}=\alpha_{j i}$ (for all $i, j \in\{1, \ldots, n\}$ ) and such a 2-form $\alpha$ is called the symmetric 2-form. We write it by $\beta_{i j} \mathrm{~d} x^{i} \odot \mathrm{d} x^{j}$ where $i \leq j, \beta_{i i}=\alpha_{i i}$ and $\beta_{i j}=2 \alpha_{i j}$ for $i \neq j$.

Analogously, the requirement $\alpha\left(\mathbf{v}_{1}, \mathbf{v}_{2}\right)=-\alpha\left(\mathbf{v}_{2}, \mathbf{v}_{1}\right)$ (for all $\mathbf{v}_{1}, \mathbf{v}_{2} \in \mathcal{V}$ ) means $\alpha_{i j}=-\alpha_{j i}$ (for all $i, j \epsilon$ $\{1, \ldots, n\}$ ) and such a 2-form $\alpha$ is called the antisymmetric (or skew-symmetric) 2-form. We write it by $\beta_{i j} \mathrm{~d} x^{i} \wedge \mathrm{d} x^{j}$ where $i<j, \beta_{i j}=\alpha_{i j}$.

In general, symmetric $k$-forms are defined by

$$
\alpha\left(\mathbf{v}_{1}, \ldots \mathbf{v}_{k}\right)=\alpha\left(\mathbf{v}_{\sigma(1)}, \ldots \mathbf{v}_{\sigma(k)}\right)
$$

for all permutations $\sigma:\{1, \ldots, k\} \rightarrow\{1, \ldots, k\}$ and for all $\mathbf{v}_{1}, \ldots, \mathbf{v}_{k} \in \mathcal{V}$. In coordinates, it yields

$$
\alpha_{i_{1} \ldots i_{k}}=\alpha_{\sigma\left(i_{1}\right) \ldots \sigma\left(i_{k}\right)}
$$

for all $i_{1}, \ldots i_{k} \in\{1, \ldots, n\}$. All symmetric $k$-forms form a vector subspace $\odot^{k}(\mathcal{V})$ of the vector space of all $k$-forms on $\mathcal{V}$, elements of it are $\beta_{i_{1} \ldots i_{k}} \mathrm{~d} x^{i_{1}} \odot \cdots \odot \mathrm{d} x^{i_{k}}$ with $i_{1} \leq \cdots \leq i_{k}$ and if $s$ is the number of permutations (with repetition) of $i_{1}, \ldots, i_{k}$, then $\beta_{i_{1} \ldots i_{k}}=s \alpha_{i_{1} \ldots i_{k}}$. Antisymmetric $k$-forms are defined by

$$
\alpha\left(\mathbf{v}_{1}, \ldots \mathbf{v}_{k}\right)=\operatorname{sgn} \sigma \alpha\left(\mathbf{v}_{\sigma(1)}, \ldots \mathbf{v}_{\sigma(k)}\right)
$$

for all permutations $\sigma:\{1, \ldots, k\} \rightarrow\{1, \ldots, k\}$ and for all $\mathbf{v}_{1}, \ldots, \mathbf{v}_{k} \in \mathcal{V}$. In coordinates, it yields

$$
\alpha_{i_{1} \ldots i_{k}}=\operatorname{sgn} \sigma \alpha_{\sigma\left(i_{1}\right) \ldots \sigma\left(i_{k}\right)}
$$


for all $i_{1}, \ldots i_{k} \in\{1, \ldots, n\}$. All antisymmetric $k$-forms form a vector subspace $\Lambda^{k}(\mathcal{V})$ of the vector space of all $k$-forms on $\mathcal{V}$, elements of it are $\beta_{i_{1} \ldots i_{k}} \mathrm{~d} x^{i_{1}} \wedge \cdots \wedge \mathrm{d} x^{i_{k}}$ with $i_{1}<\cdots<i_{k}$ and $\beta_{i_{1} \ldots i_{k}}=s \alpha_{i_{1} \ldots i_{k}}$.

(Attention! Some authors, by the word "form", mean antisymmetric forms only.)

2.2. Differential $k$-forms. If we replace the vector space $\mathcal{V}$ by the space $\mathfrak{X}(M)$ of vector fields on a manifold $M$, $\operatorname{dim} M=n, k$-forms become differential $k$-forms and $\alpha_{i_{1} \ldots i_{k}}\left(x^{1}, \ldots, x^{n}\right)$ are functions on $M$.

(As above, some authors consider antisymmetric differential $k$-forms only.)

The space of all antisymmetric differential $k$-forms on a manifold $M$ is denoted by $\Omega^{k}(M)$.

Let us also note here - which will be useful in the text below - that a permutation $\sigma:\{1, \ldots, k, k+1, \ldots, k+l\} \rightarrow$ $\{1, \ldots, k, k+1, \ldots, k+l\}$ satisfying $\sigma(1)<\cdots<\sigma(k)$ and $\sigma(k+1)<\cdots<\sigma(k+l)$ is called $(k, l)$-shuffle.

2.3. Three operations on antisymmetric (differential) $k$-forms. The following three operations have remarkable properties and are related to each other. But we will focus mainly on calculations.

2.3.1. The wedge product. The wedge product is a binary operation

$$
\wedge: \Lambda^{k}(\mathcal{V}) \times \Lambda^{l}(\mathcal{V}) \rightarrow \Lambda^{k+l}(\mathcal{V})
$$

or (without any changes)

$$
\wedge: \Omega^{k}(M) \times \Omega^{l}(M) \rightarrow \Omega^{k+l}(M)
$$

defined by

$$
\gamma=\alpha \wedge \beta ; \quad \gamma_{i_{1} \ldots i_{k+l}}=\sum_{\sigma} \operatorname{sgn} \sigma \alpha_{i_{\sigma(1)} \ldots i_{\sigma(k)}} \beta_{i_{\sigma(k+1)} \ldots i_{\sigma(k+l)}},
$$

where $\sigma$ represent $(k, l)$-shuffles.

Example 1. First, something absolutely trivial. Let $n=2, \alpha=\mathrm{d} x, \beta=\mathrm{d} y$ and let us "compute" $\gamma=\alpha \wedge \beta$.

We have $\alpha_{1}=\beta_{2}=1$ and $\alpha_{2}=\beta_{1}=0$. Then

$$
\gamma_{12}=\alpha_{1} \beta_{2}-\alpha_{2} \beta_{1}=1
$$

it follows

$$
\gamma=\mathrm{d} x \wedge \mathrm{d} y
$$

Example 2. Of course, the product $\alpha \wedge \alpha$ need not be zero. Let $n=4, \alpha=\mathrm{d} x^{1} \wedge \mathrm{d} x^{2}+\mathrm{d} x^{3} \wedge \mathrm{d} x^{4}$ and let us compute $\gamma=\alpha \wedge \alpha$.

We have $\alpha_{12}=\alpha_{34}=1$ and $\alpha_{13}=\alpha_{14}=\alpha_{23}=\alpha_{23}=0$. Then

$$
\gamma_{1234}=\alpha_{12} \alpha_{34}-\alpha_{13} \alpha_{24}+\alpha_{14} \alpha_{23}+\alpha_{23} \alpha_{14}-\alpha_{24} \alpha_{13}+\alpha_{34} \alpha_{12}=2
$$

it follows

$$
\gamma=2 \mathrm{~d} x^{1} \wedge \mathrm{d} x^{2} \wedge \mathrm{d} x^{3} \wedge \mathrm{d} x^{4}
$$

Example 3. Let us look at differential forms, too. Let $n=3, \alpha=(x+y) \mathrm{d} y+\left(x^{2}-y^{2}\right) \mathrm{d} z, \beta=z \mathrm{~d} x \wedge \mathrm{d} y+x z \mathrm{~d} z$ and let us compute $\gamma=\alpha \wedge \alpha$. 
We have $\alpha_{2}=x+y, \alpha_{3}=x^{2}-y^{2}, \beta_{12}=z, \beta_{13}=x z$ and $\alpha_{1}=\beta_{23}=0$. Then

$$
\gamma_{123}=\alpha_{1} \beta_{23}-\alpha_{2} \beta_{13}+\alpha_{3} \beta_{12}=-x y z-y^{2} z,
$$

it follows

$$
\gamma=\left(-x y z-y^{2} z\right) \mathrm{d} x \wedge \mathrm{d} y \wedge \mathrm{d} z=\left(x y z+y^{2} z\right) \mathrm{d} x \wedge \mathrm{d} z \wedge \mathrm{d} y .
$$

2.3.2. The exterior derivative. The exterior derivative is a unary operation

$$
\mathrm{d}: \Omega^{k}(M) \rightarrow \Omega^{k+l}(M)
$$

defined by

$$
\mathrm{d}(\alpha)=\mathrm{d} \alpha ; \quad \mathrm{d}\left(\alpha_{i_{1} \ldots i_{k}} \mathrm{~d} x^{i_{1}} \wedge \cdots \wedge \mathrm{d} x^{i_{k}}\right)=\left(\frac{\partial \alpha_{i_{1} \ldots i_{k}}}{\partial x^{i}} \mathrm{~d} x^{i}\right) \wedge \mathrm{d} x^{i_{1}} \wedge \cdots \wedge \mathrm{d} x^{i_{k}}
$$

(Einstein summation convention in brackets!).

Example 4. Let $n=3, \alpha=\left(x+z^{2}\right) \mathrm{d} x \wedge \mathrm{d} y+\left(x+y+z^{2}\right) \mathrm{d} x \wedge \mathrm{d} z$. We have $\alpha_{12}=x+z^{2}, \alpha_{13}=x+y+z^{2}, \alpha_{23}=0$.

Then

$$
\begin{aligned}
\mathrm{d} \alpha= & (\mathrm{d} x+2 z \mathrm{~d} z) \wedge \mathrm{d} x \wedge \mathrm{d} y+(\mathrm{d} x+\mathrm{d} y+2 z \mathrm{~d} z) \wedge \mathrm{d} x \wedge \mathrm{d} z= \\
& 2 z \mathrm{~d} z \wedge \mathrm{d} x \wedge \mathrm{d} y+\mathrm{d} y \wedge \mathrm{d} x \wedge \mathrm{d} z=(2 z-1) \mathrm{d} x \wedge \mathrm{d} y \wedge \mathrm{d} z
\end{aligned}
$$

Example 5. For 0-forms, i.e. functions, we obtain the well-known differential. Let $n=3, \alpha=2 x y+z^{3}$.

Then

$$
\mathrm{d} \alpha=2 y \mathrm{~d} x+2 x \mathrm{~d} y+3 z^{2} \mathrm{~d} z
$$

2.3.3. The Hodge star operator. The Hodge star operator is a unary operation

$$
\star: \Lambda^{k}(\mathcal{V}) \rightarrow \Lambda^{n-k}(\mathcal{V})
$$

or (without any changes)

$$
\star: \Omega^{k}(M) \rightarrow \Omega^{n-k}(M),
$$

where $n=\operatorname{dim} \mathcal{V}$ or $n=\operatorname{dim} M$.

For this operator, however, it is necessary to assume that an inner product is given on the vector space $\mathcal{V}$ (or, on tangent spaces $T_{x} M$ in each $\left.x \in M\right)$. Due to this inner product, we have an orthonormal basis $\mathbf{e}_{1}, \ldots, \mathbf{e}_{n}$.

The Hodge star operator is defined by the assigning of a basis of $\Lambda^{n-k}(\mathcal{V})$ to the basis of $\Lambda^{k}(\mathcal{V})$ by the following way

$$
\star: \mathbf{e}_{\sigma(1)} \wedge \mathbf{e}_{\sigma(k)} \longmapsto e_{\sigma(k+1)} \wedge \mathbf{e}_{\sigma(n)}
$$

for every $(k, n-k)$-shuffle $\sigma$. This is naturally extended to the entire vector space $\mathcal{V}$.

Example 6. Let $n=3, k=2$. Then

$$
\mathbf{e}_{1} \wedge \mathbf{e}_{2} \longmapsto \mathbf{e}_{3}, \quad \mathbf{e}_{1} \wedge \mathbf{e}_{3} \longmapsto-\mathbf{e}_{2}, \quad \mathbf{e}_{2} \wedge \mathbf{e}_{3} \longmapsto \mathbf{e}_{1}
$$


Then for $\mathbf{v}_{1}=v_{1}^{i} \mathbf{e}_{i}, \mathbf{v}_{2}=v_{2}^{i} \mathbf{e}_{i}$ we have $\mathbf{v}_{1} \wedge \mathbf{v}_{2}=v_{1}^{i} v_{2}^{j} \mathbf{e}_{i} \wedge \mathbf{e}_{j}$ and

$$
\begin{aligned}
\star & \left(\mathbf{v}_{1} \wedge \mathbf{v}_{2}\right)=v_{1}^{i} v_{2}^{j} \star\left(\mathbf{e}_{i} \wedge \mathbf{e}_{j}\right)= \\
& \left(v_{1}^{1} v_{2}^{2}-v_{1}^{2} v_{2}^{1}\right) \mathbf{e}_{3}+\left(v_{1}^{1} v_{2}^{3}-v_{1}^{3} v_{2}^{1}\right)\left(-\mathbf{e}_{2}\right)+\left(v_{1}^{2} v_{2}^{3}-v_{1}^{3} v_{2}^{2}\right) \mathbf{e}_{1}
\end{aligned}
$$

which is nothing but the usual vector product.

2.4. The split of a $k$-form. The linear operator $\lrcorner$ is precisely defined e.g. in the book [4] Lectures on Differential Geometry by S. Sternberg, Chapter I.4. We simplify the definition by $\mathbf{v}\lrcorner \omega=\omega(\mathbf{v}, \ldots)$. Then for any $k$-form $\omega \in \Lambda^{k}(\mathcal{V}), \alpha \in \Lambda^{1}(\mathcal{V})$ and $\mathbf{v} \in \mathcal{V}$ the formula

$$
\mathbf{v}\lrcorner(\alpha \wedge \omega)=\alpha(\mathbf{v}) \cdot \omega
$$

holds and, of course, the analogous claim holds for differential forms, too. We present it in the following example.

Example 7. Let $n=3, k=2$. Then $\mathbf{v}=v^{1} \frac{\partial}{\partial x}+v^{2} \frac{\partial}{\partial y}+v^{3} \frac{\partial}{\partial z}, \alpha=\alpha_{1} \mathrm{~d} x+\alpha_{2} \mathrm{~d} y+\alpha_{3} \mathrm{~d} z$ and $\omega=\omega_{12} \mathrm{~d} x \wedge \mathrm{d} y-\omega_{13} \mathrm{~d} x \wedge$ $\mathrm{d} z+\omega_{23} \mathrm{~d} y \wedge \mathrm{d} z$. Then

$$
\alpha \wedge \omega=\alpha_{1} \omega_{23} \mathrm{~d} x \wedge \mathrm{d} y \wedge \mathrm{d} z+\alpha_{2} \omega_{13} \mathrm{~d} y \wedge \mathrm{d} x \wedge \mathrm{d} z+\alpha_{3} \omega_{12} \mathrm{~d} z \wedge \mathrm{d} x \wedge \mathrm{d} y
$$

and

$$
\begin{aligned}
\mathbf{v}\lrcorner(\alpha \wedge \omega)= & \alpha_{1} \omega_{23} v^{1} \mathrm{~d} y \wedge \mathrm{d} z-\alpha_{1} \omega_{23} v^{2} \mathrm{~d} x \wedge \mathrm{d} z+\alpha_{1} \omega_{23} v^{3} \mathrm{~d} x \wedge \mathrm{d} y+ \\
& \alpha_{2} \omega_{13} v^{1} \mathrm{~d} y \wedge \mathrm{d} z-\alpha_{2} \omega_{13} v^{2} \mathrm{~d} x \wedge \mathrm{d} z+\alpha_{2} \omega_{13} v^{3} \mathrm{~d} x \wedge \mathrm{d} y+ \\
& \alpha_{3} \omega_{12} v^{1} \mathrm{~d} y \wedge \mathrm{d} z-\alpha_{3} \omega_{12} v^{2} \mathrm{~d} x \wedge \mathrm{d} z+\alpha_{3} \omega_{12} v^{3} \mathrm{~d} x \wedge \mathrm{d} y \\
= & \left(\alpha_{1} \omega_{23} v^{3}+\alpha_{2} \omega_{13} v^{3}+\alpha_{3} \omega_{12} v^{3}\right) \mathrm{d} x \wedge \mathrm{d} y- \\
& \left(\alpha_{1} \omega_{23} v^{2}+\alpha_{2} \omega_{13} v^{2}+\alpha_{3} \omega_{12} v^{2}\right) \mathrm{d} x \wedge \mathrm{d} z+ \\
& \left(\alpha_{1} \omega_{23} v^{1}+\alpha_{2} \omega_{13} v^{1}+\alpha_{3} \omega_{12} v^{1}\right) \mathrm{d} y \wedge \mathrm{d} z .
\end{aligned}
$$

Further,

$$
\begin{aligned}
\mathbf{v}\lrcorner \omega & =\omega_{12} v^{1} \mathrm{~d} y-\omega_{12} v^{2} \mathrm{~d} x-\omega_{13} v^{1} \mathrm{~d} z+\omega_{13} v^{3} \mathrm{~d} x+\omega_{23} v^{2} \mathrm{~d} z-\omega_{23} v^{3} \mathrm{~d} y \\
& =\left(-\omega_{12} v^{2}+\omega_{13} v^{3}\right) \mathrm{d} x+\left(\omega_{12} v^{1}-\omega_{23} v^{3}\right) \mathrm{d} y+\left(-\omega_{13} v^{1}+\omega_{23} v^{2}\right) \mathrm{d} z
\end{aligned}
$$

and

$$
\begin{aligned}
\alpha \wedge(\mathbf{v}\lrcorner \omega)= & \left(\alpha_{1}\left(\omega_{12} v^{1}-\omega_{23} v^{3}\right)-\alpha_{2}\left(-\omega_{12} v^{2}+\omega_{13} v^{3}\right)\right) \mathrm{d} x \wedge \mathrm{d} y- \\
& \left(\alpha_{1}\left(-\omega_{13} v^{1}+\omega_{23} v^{2}\right)-\alpha_{3}\left(-\omega_{12} v^{2}+\omega_{13} v^{3}\right)\right) \mathrm{d} x \wedge \mathrm{d} z+ \\
& \left(\alpha_{2}\left(-\omega_{13} v^{1}+\omega_{23} v^{2}\right)-\alpha_{3}\left(\omega_{12} v^{1}-\omega_{23} v^{3}\right)\right) \mathrm{d} y \wedge \mathrm{d} z .
\end{aligned}
$$


Now, we have

$$
\begin{aligned}
& \mathbf{v}\lrcorner(\alpha \wedge \omega)+\alpha \wedge(\mathbf{v}\lrcorner \omega)= \\
& \left(\alpha_{1} \omega_{12} v^{1}+\alpha_{2} \omega_{12} v^{2}+\alpha_{3} \omega_{12} v^{3}\right) \mathrm{d} x \wedge \mathrm{d} y+ \\
& \left(-\alpha_{1} \omega_{13} v^{1}-\alpha_{2} \omega_{13} v^{2}-\alpha_{3} \omega_{13} v^{3}\right) \mathrm{d} x \wedge \mathrm{d} z+ \\
& \left(\alpha_{1} \omega_{23} v^{1}+\alpha_{2} \omega_{23} v^{2}+\alpha_{3} \omega_{23} v^{3}\right) \mathrm{d} y \wedge \mathrm{d} z= \\
& \alpha(\mathbf{v}) \cdot \omega .
\end{aligned}
$$

Acknowledgement: The author was supported by Brno University of Technology, the specific research plan No. FSI-S-17-4464.

\section{REFERENCES}

1. J. Kocik, Relativistic observer and Maxwell's equations: an example of a non-principal Ehresmann connection, arXiv:1604.00081v1 [math-ph], 2016.

2. I. Kolář, P. W. Michor J. and Slovák, Natural Operations in Differential Geometry, Springer Verlag, 1993.

3. M. Olive, Effective computation of $\mathrm{SO}(3)$ and $\mathrm{O}(3)$ linear representation symmetry classes, arXiv:1709.01776v3 [math.RT], 2018.

4. S. Sternberg, Lectures on Differential Geometry, Vol. 316, Amer. Math. Soc., 1999. 